\title{
Nanoscale
}

Check for updates

Cite this: Nanoscale, 2018, 10, 6270

Received 1st December 2017.

Accepted 6th March 2018

DOI: 10.1039/c7nr08979a

rsc.li/nanoscale

\section{Large-scale dewetting assembly of gold nanoparticles for plasmonic enhanced upconversion nanoparticles $\uparrow$}

\author{
Christian Clarke, (iD) a Deming Liu, ${ }^{\mathrm{b}}$ Fan Wang, (iD a Yongtao Liu, ${ }^{a}$ Chaohao Chen, (iD) a \\ Cuong Ton-That, ${ }^{a}$ Xiaoxue $\mathrm{Xu}^{\star \mathrm{a}}$ and Dayong Jin (D) *a,c
}

\begin{abstract}
Plasmonic nanostructures have been broadly investigated for enhancing many photophysical properties of luminescent nanomaterials. Precisely controlling the distance between the plasmonic nanostructure and the luminescent material is challenging particularly for the large-scale production of individual nanoparticles. Here we report an easy and reliable method for the large-scale dewetting of plasmonic gold nanoparticles onto core-shell (CS) upconversion nanoparticles (UCNPs). A commensurate $\mathrm{NaYF}_{4}$ shell with a thickness between $5 \mathrm{~nm}$ and $15 \mathrm{~nm}$ is used as a tunable spacer to control the distance between the UCNP and the plasmonic gold nanoparticles. The upconversion emission intensity of single gold decorated core-inert shell (Au-CS) UCNPs is quantitatively characterized using a scanning confocal microscope. The results demonstrate the highest feasible enhancement of upconversion emission and a record reduction in lifetime for UCNPs fabricated in this manner. The Au-CS UCNPs are further investigated by simulation and synchrotron near edge X-ray absorption fine structure (NEXAFS) analysis.
\end{abstract}

\section{Introduction}

The sequential absorption of two or more low energy photons to emit one higher energy photon is the process referred to as photon upconversion. ${ }^{1}$ The current upconversion research is focused on developing upconversion nanoparticles (UCNPs), ${ }^{2}$ with sizes in the range of 5 to $100 \mathrm{~nm}$ for applications such as

\footnotetext{
${ }^{a}$ Institute for Biomedical Materials and Devices, Faculty of Science, University of Technology Sydney, NSW, 2007, Australia. E-mail: dayong.jin@uts.edu.au, xiaoxuehelen.xu@uts.edu.au,cuong.ton-that@uts.edu.au

${ }^{b}$ Department of Chemistry and Biochemistry, Concordia University, Montréal, QC H4B 1R6, Canada

${ }^{c}$ ARC Research Hub for Integrated Device for End-user Analysis at Low-levels (IDEAL), Faculty of Science, University of Technology Sydney, NSW, 2007, Australia

$\dagger$ Electronic supplementary information (ESI) available: General procedures, simulation details, additional TEM/SEM images of the synthesized materials, simulation results, additional NEXAFS/XRD spectra, additional scanning confocal images and an optical path diagram of the confocal scanning microscope. See DOI: $10.1039 / \mathrm{c} 7$ nro8979a
}

bioimaging, ${ }^{3,4}$ biosensing, ${ }^{5}$ nanoscopy, theranostics, anticounterfeiting, displays and photovoltaics. ${ }^{6}$ One of the most efficient UCNP materials, lanthanide ion $\left(\mathrm{Ln}^{3+}\right)$ doped $\beta-\mathrm{NaYF}_{4}$ host nanocrystals, still suffers a low quantum yield $(\phi<5 \%){ }^{7}$ which is far lower than the theoretical value for the twophoton process of upconversion $(\phi=50 \%),{ }^{8}$ limiting the practical applications of UCNPs.

To address this issue, a number of methods have been applied to enhance upconversion emission including host lattice manipulation, surface passivation, energy transfer modulation, broadband sensitization, surface plasmon coupling and photonic crystal engineering. ${ }^{9}$ Plasmonic nanoparticles of noble metals such as gold $(\mathrm{Au})$ and silver $(\mathrm{Ag})$ with tunable visible light absorption and scattering are frequently used to couple with UCNPs to enhance their upconversion emission. This plasmonic enhancement is typically dependent on the size, shape and composition of the noble metal nanoparticles used as well as the distance between the UCNPs and noble metal nanoparticles. ${ }^{10,11}$ These factors have led to both $\mathrm{Au}$ and $\mathrm{Ag}$ being used for plasmonic coupling in the forms of spherical nanoparticles, ${ }^{12}$ nanowires,${ }^{13}$ nanoislands ${ }^{14}$ and lithographically patterned nanoarrays. ${ }^{15}$

In this work, $\mathrm{Au}$ is easily produced into uniform spherical nanoparticles by sputtering, thermal annealing and dewetting. Dewetting is the disaggregation of a thin film into discrete particles, mediated by a minimisation of surface energy. ${ }^{16}$ This dewetting method allows the shape and size of the Au nanoparticles to be precisely controlled on a large scale so that their plasmon resonance can be tuned to match the emission wavelength of $\mathrm{Er}^{3+}$ emitters in $\mathrm{NaYF}_{4} \mathrm{UCNPs}^{17}$ by simply varying the film thickness, annealing temperature and annealing time. ${ }^{18,19}$ Secondarily, the annealing during the dewetting of the $\mathrm{Au}$ onto the surface of the CS UCNPs promotes the diffusion of $\mathrm{Ln}^{3+}$ dopants within the UCNPs, reducing internal crystal defects which can lead to an increased quantum efficiency. ${ }^{20}$

The design, consisting of an optically active UCNP core, an inert shell spacer and satellite plasmonic Au nanoparticles decorated on the shell, is chosen to suit the dewetting method 
for a large-scale assembly of Au nanoparticles onto single and mono-dispersed hybrid CS UCNPs. Fundamental to this design is the spacer which is vital for minimising the nonradiative energy transfer which causes luminescence quenching due to the direct contact of the UCNPs with the Au nanoparticle surface. ${ }^{12}$

From this design, similar structures have been previously fabricated including both metal core-spacer-UC shell nanoparticles $^{21-23}$ and UC core-spacer-metal shell nanoparticles $^{24,25}$ as summarised in Table S1. $\dagger$ However, these previous studies relied exclusively on a spacer of metal, polymer or $\mathrm{SiO}_{2}$ for attaching the Au nanoparticles by solution processing, which inhibits the plasmonic enhancement of luminescence due to the apparent parasitic absorption by these materials at the thicknesses required for optimum enhancement. ${ }^{26,27}$

This limitation is overcome in this work by a third enhancement method using an undoped inert crystal shell. The thickness of inert shells is highly controllable and can be varied, allowing nanometre control by adjusting the concentration and amount of the shell precursor added during the epitaxial growth. ${ }^{28-30}$ Inert shells of the host crystal $\left(\mathrm{NaYF}_{4}\right.$ devoid of $\mathrm{Ln}^{3+}$ ) are employed to achieve both a reduction in surface defects to enhance the luminescence ${ }^{31}$ and functionality as the spacer in the design. To the authors' knowledge, this integrated method constitutes the first combination of core-shell UCNPs, annealing and plasmonic enhancement in a single process to produce a singularly enhanced UCNP.

\section{Results and discussion}

Fig. 1(A) shows the TEM images of the core UCNPs with an average diameter of $25 \mathrm{~nm}$. The core UCNPs are well-dispersed nanoparticles with a highly morphological uniformity. Fig. 1 (B) and Fig. S1(A \& C) $\uparrow$ show the CS UCNPs with an inert $\mathrm{NaYF}_{4}$ shell coating of thicknesses $5 \mathrm{~nm}, 10 \mathrm{~nm}$ and $15 \mathrm{~nm}$ respectively; thus, the formed uniform CS UCNPs are of sizes $35 \mathrm{~nm}, 45 \mathrm{~nm}$ and $55 \mathrm{~nm}$. The ellipsoid shape of the $35 \mathrm{~nm}$ CS UCNPs is due to the longitudinal preference of the epitaxial growth, which is especially pronounced when thin shell coatings are grown. This variation in the UCNP diameter of the near-spherical UCNPs is within $2 \mathrm{~nm}$, significantly smaller than the incremental step $(5 \mathrm{~nm})$ in the varied shell thickness. The dewetted Au nanoparticles shown in Fig. S1(B) $\dagger$ are uniformly scattered on the substrate having an average size of $15 \mathrm{~nm}$. The Au-CS UCNPs in Fig. 1(C) show the typical morphology of the dewetted Au onto the surface of CS UCNPs. The CS UCNPs have not undergone any morphology change while the Au nanoparticles have directly dewetted onto the surface of the UCNPs and the desired structure of Au-CS UCNPs is produced. Fig. S1(D) $\dagger$ shows an additional image of the same sample as shown in Fig. 1(C) of the Au-CS UCNPs, and it highlights that the method can produce well-dispersed single $\mathrm{Au}-$ CS UCNPs. The far separated CS UCNPs coated with Au can be used directly for single nanoparticle luminescence measure-

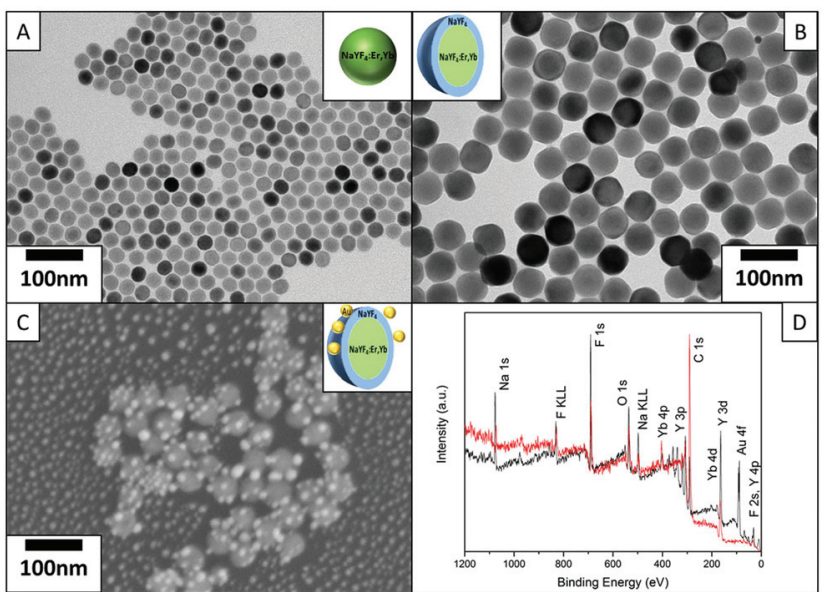

Fig. 1 TEM image of $25 \mathrm{~nm}$ diameter core UCNPs of $\beta-\mathrm{NaYF}_{4}: 20 \%$ $\mathrm{Yb}, 2 \% \operatorname{Er}(\mathrm{A}), 55 \mathrm{~nm}$ diameter CS UCNPs of $\beta-\mathrm{NaYF}_{4}: 20 \% \quad \mathrm{Yb}, 2 \%$ $\mathrm{Er}\left(\mathrm{NaYF}_{4}\right.$ (B), SEM image of assembly of Au-CS UCNPs of $55 \mathrm{~nm}$ diameter $\beta-\mathrm{NaYF}_{4}: 20 \% \quad \mathrm{Yb}, 2 \% \quad$ EraNaYF 4 after annealing at $400{ }^{\circ} \mathrm{C}$ for 1 hour (C) and XPS survey spectra of $55 \mathrm{~nm}$ diameter CS UCNPs (red) and the assembled Au-CS UCNPs of $55 \mathrm{~nm}$ diameter after annealing (black) (D).

ment. XPS survey spectra of both $55 \mathrm{~nm}$ CS UCNPs and the asobtained $\mathrm{Au}-\mathrm{CS}$ UCNPs after dewetting in Fig. 1(D) confirm the composition of the samples. The presence of $\mathrm{Na}, \mathrm{Y}, \mathrm{F}$ and $\mathrm{Yb}$ in both spectra can be attributed to the CS UCNPs and the $\mathrm{C}$ and $\mathrm{O}$ peaks result from adventitious carbon. ${ }^{32}$ The additional Au peak is only present in the Au-CS UCNPs after dewetting. High surface density samples were prepared for XPS on a $\mathrm{Si}$ substrate. The absence of $\mathrm{Si}$ signals in both spectra indicates that XPS signals arise from the UCNP films with no contributions from the silicon substrate. As a result, the entire Au signal is detected from the dewetted Au nanoparticles on the CS UCNPs rather than Au dewetted on the surface of the substrate.

The dewetting treatment at $400{ }^{\circ} \mathrm{C}$ for 1 hour is not only crucial to convert the Au film into discrete nanoparticles of specific size $^{18}$ on the CS UCNPs surface, but it is also the optimum condition to prevent the CS UCNPs from aggregating and changing the phase. The XRD characterization in Fig. S2 (C) $\dagger$ on the CS UCNPs before and after annealing at $400{ }^{\circ} \mathrm{C}$ for 1 hour shows that the CS UCNPs retain their $\beta$ phase. ${ }^{33,34}$

To investigate the effect of annealing on the diffusion of $\mathrm{Er}$ and $\mathrm{Yb}$ dopants within the CS UCNPs, Near Edge X-ray Absorption Fine Structure (NEXAFS) analysis was conducted at the Australia Synchrotron. NEXAFS analysis in partial electron yield (PEY) and total fluorescent yield (TFY) using a modified procedure $^{35}$ was performed to measure the re-distribution of the Er and Yb dopants in the CS UCNPs due to the annealing process. Fig. 2(A \& C) shows NEXAFS spectra in TFY mode which probes the entire UCNP volume. The TFY intensity for both Er (Fig. 2A) and Yb (Fig. 2C) before and after annealing is the same, and this indicates that for both samples, the surface coverage of UCNPs on the substrate is approximately equal. In PEY mode, the NEXAFS spectra are being primarily generated 

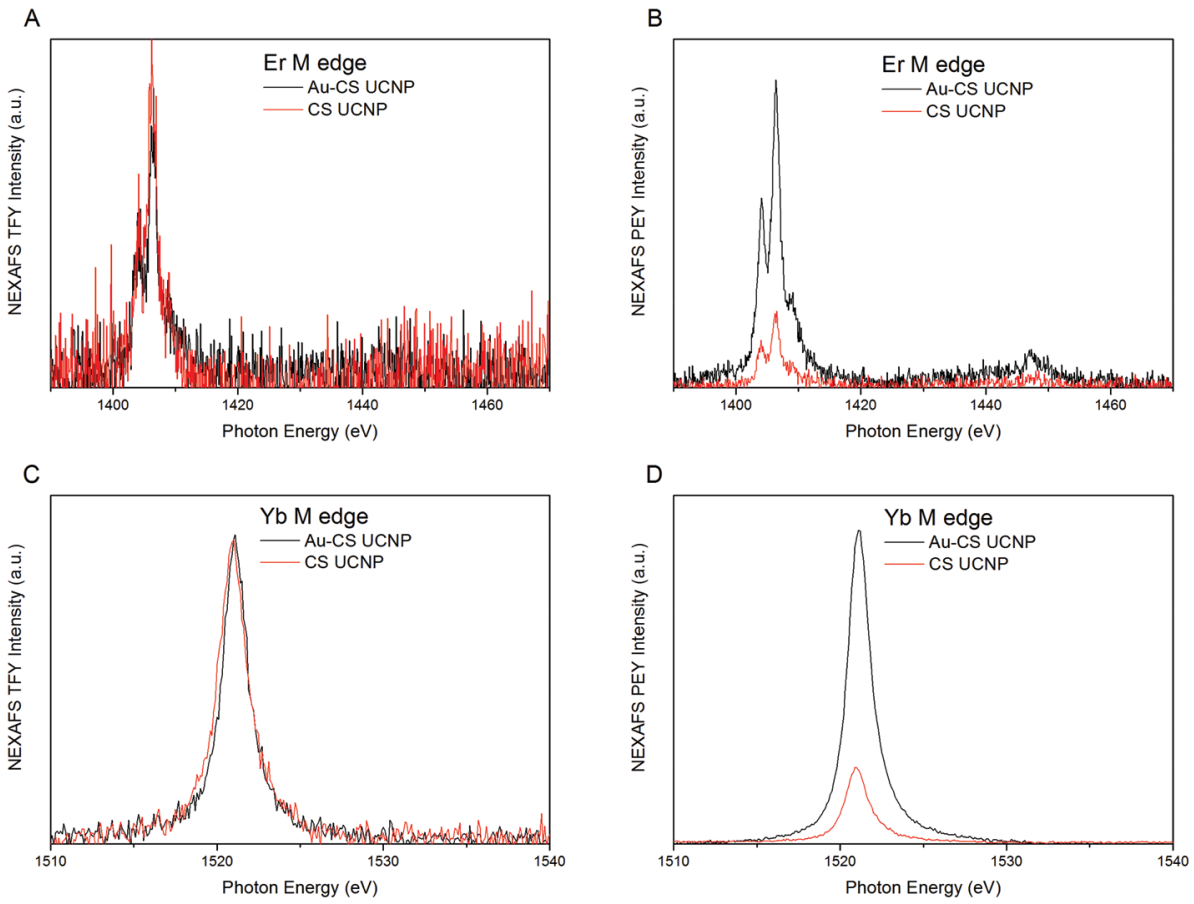

Fig. 2 NEXAFS spectra for Er M edge and Yb M edge of the CS UCNPs with a $5 \mathrm{~nm}$ shell before (CS UCNP) and after annealing (Au-CS UCNP) in TFY $(A \& C)$ and PEY $(B \& D)$ modes.

from only the shell volume allowing for any change in the distribution of the dopants to be identified. For both Er (Fig. 2B) and $\mathrm{Yb}$ (Fig. 2D) after annealing, there is an increase in the intensity in the PEY spectrum. This suggests a denser Yb and Er dopant distribution towards the surface of the CS UCNPs after annealing, indicating that both dopants have diffused further from the core towards the inert shell during the annealing process. The driving force for the diffusion would be the concentration gradient of the dopant from the core nanocrystal to the inert shell accelerated under the annealing conditions. As the surface coverage was approximately equal, the relative increase of dopants diffused towards the shell after annealing can be estimated to be a factor of 3.8 and 4.4 for $\mathrm{Er}$ and $\mathrm{Yb}$, respectively. As expected, the increase in $\mathrm{Yb}$ is higher than $\mathrm{Er}$ due to the higher concentration gradient of $\mathrm{Yb}$ to $\operatorname{Er}(20 \%$ to $2 \%$ ) across the CS UCNPs. The same result was demonstrated by the CS UCNPs and Au-CS UCNPs with a $15 \mathrm{~nm}$ shell. The results are shown in Fig. $\mathrm{S} 2, \uparrow$ in which a relative increase in $\mathrm{Yb}$ after annealing is estimated to be a factor of 1.9.

The diffusion of the dopant ions from the core UCNPs to the inert shell firstly produces a reduced effective distance between the $\mathrm{Au}$ nanoparticles on the inert shell and the active core UCNPs. Concurrently, this diffusion also confirms that the annealing has facilitated an increase in the diffusion coefficient of the $\mathrm{Ln}^{3+}$ dopants producing an improved and refined crystal from internal defect removal during diffusion. ${ }^{20,34}$

To study the surface plasmon resonance of the Au-CS UCNPs system, the luminescent emission of CS UCNPs and the optical transmission of the dewetted $\mathrm{Au}$ nanoparticles were characterized. As shown in Fig. 3(A), the Au nanoparticles of size $15 \mathrm{~nm}$ produced from dewetting show a broad absorption peaked at $540 \mathrm{~nm}$. The luminescent emission of the CS UCNPs shows the green emission at $525 \mathrm{~nm}$ and $540 \mathrm{~nm}$ and the red emission at $650 \mathrm{~nm}$ and $660 \mathrm{~nm}$. The surface plasmon resonance of the $\mathrm{Au}$ nanoparticles overlaps precisely with the green emissions which are the radiative transitions of $\left({ }^{2} \mathrm{H}_{11 / 2},{ }^{4} \mathrm{~S}_{3 / 2}\right) \rightarrow{ }^{4} \mathrm{I}_{15 / 2}$ of $\mathrm{Er}^{3+}$ from the UCNPs as shown in Fig. 3(B).

To investigate the luminescence performance of the $\mathrm{Au}-\mathrm{CS}$ UCNPs, the emission intensity of single CS UCNPs, annealed CS UCNPs and assembled Au-CS UCNPs were recorded using a purpose-built scanning confocal microscopy system for single nanoparticles. ${ }^{36,37}$ The scanned images of Fig. 4 show the single nanoparticle emission images of the core UCNPs (A), CS UCNPs with a $5 \mathrm{~nm}, 10 \mathrm{~nm}$ and $15 \mathrm{~nm}$ inert shell (B-D), core Au-UCNPs (E), and Au-CS UCNPs with a $5 \mathrm{~nm}$, $10 \mathrm{~nm}$ and $15 \mathrm{~nm}$ inert shell $(\mathrm{F}-\mathrm{H})$, respectively. Fig. S3† additionally shows the annealed CS UCNPs scanned images of the core UCNPs (A), and CS UCNPs with a $5 \mathrm{~nm}, 10 \mathrm{~nm}$ and $15 \mathrm{~nm}$ inert shell (B-D) after annealing under identical conditions but without an Au film. Single UCNPs can be reliably identified using this method due to their near identical emission intensity. The summary of these emission intensity measurements in Fig. 4(I) has the CS UCNPs' emission enhancement saturated at a shell thickness of $5 \mathrm{~nm}$ and above as reported previously, ${ }^{31}$ while the luminescence intensity of the annealed CS UCNPs without Au displays an insignificant variation to the CS UCNPs with increasing shell thickness. This result is expected from the XRD results in Fig. $\mathrm{S} 2(\mathrm{C}) \dagger$ that show the 


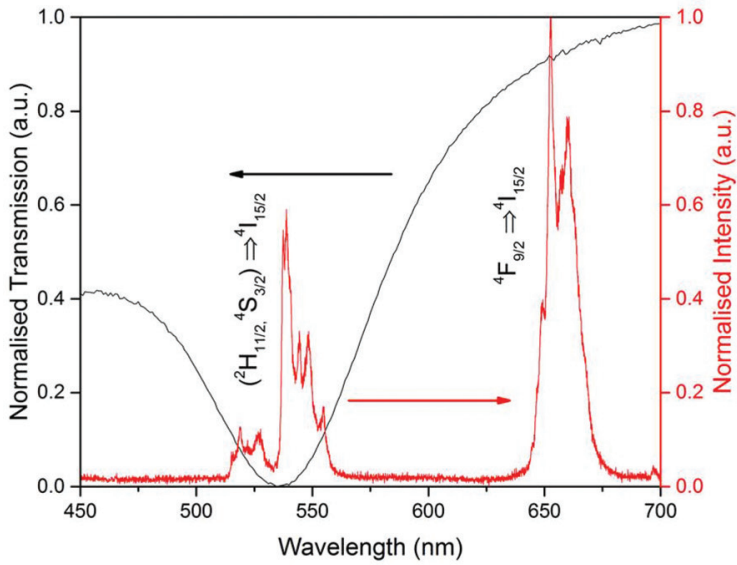

Fig. 3 Luminescence spectrum of $\beta-\mathrm{NaYF}_{4}: 20 \% \mathrm{Yb}, 2 \%$ Er UCNPs (red) overlayed with the UV-Vis transmission of pure dewetted Au nanoparticles (black) (A) and the energy level diagram of the $\mathrm{Yb}-\mathrm{Er}$ upconversion system showing the upconversion excitation and the green and red emissions with their wavelengths (B). The surface plasmon resonance $(\sim 540 \mathrm{~nm})$ of the dewetted Au nanoparticles matches the $\left({ }^{2} \mathrm{H}_{11 / 2},{ }^{4} \mathrm{~S}_{3 / 2}\right) \rightarrow{ }^{4} \mathrm{I}_{15 / 2}$ emission of $\mathrm{Er}^{3+}$
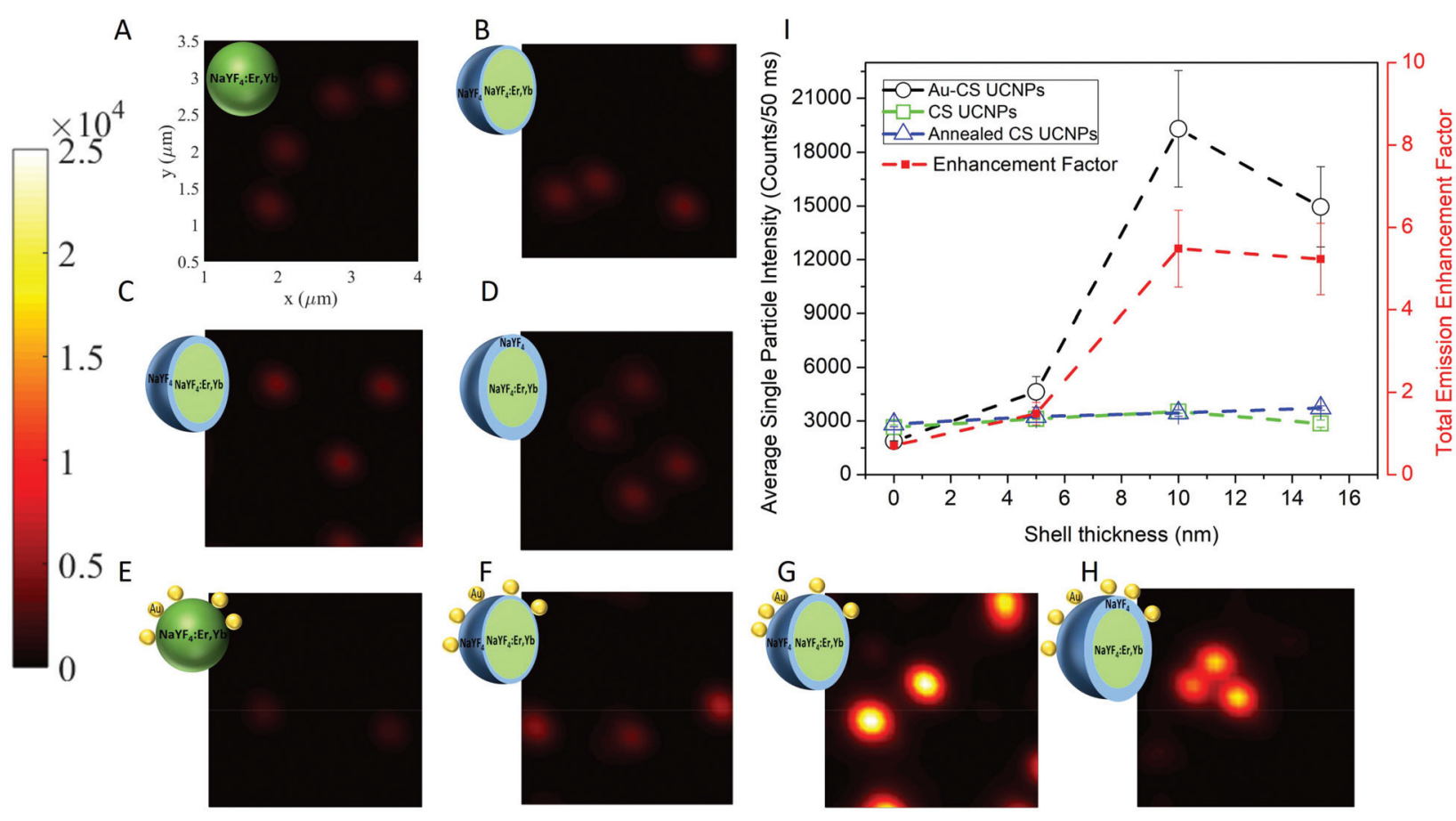

Fig. 4 The scanning confocal images of the core UCNPs (A), CS UCNPs with a $5 \mathrm{~nm}, 10 \mathrm{~nm}$ and $15 \mathrm{~nm}$ inert shell (B-D), and core Au-UCNPs (E), $\mathrm{Au}-\mathrm{CS}$ UCNPs with a $5 \mathrm{~nm}, 10 \mathrm{~nm}$ and $15 \mathrm{~nm}$ inert shell (F-H), respectively. Each scanned image is $3 \mu \mathrm{m} \times 3 \mu \mathrm{m}$ as indicated in (A) and the colour bar indicates the emission intensity range of the single UCNPs. Single UCNP total emission intensity and enhancement factor (I) for CS UCNPs (A$D), A u-C S$ UCNPs $(E-H)$ and annealed CS UCNPs (Fig. S3A-D †). The laser power was fixed at $20 \mathrm{~mW}$, which was measured at the objective back aperture.

annealed CS UCNPs retained their $\beta$ phase. It also suggests that any luminescence enhancement resulting from the improved crystal quality obtained by the reduction of internal defects during the diffusion of dopants as demonstrated by the NEXAFS results in Fig. 2 has been effectively cancelled out by the increase in the distance between the dopant $\mathrm{Yb}$ and $\mathrm{Er}$ ions this diffusion would cause. This increase in distance between the dopant ions would reduce the probability of energy transfer between $\mathrm{Yb}-\mathrm{Yb}$ and $\mathrm{Yb}$-Er reducing upconversion emission. 
On the other hand, the luminescence intensity of the CS UCNPs decorated with dewetted $\mathrm{Au}$ nanoparticles (Au-CS UCNPs) demonstrates a clear dependence on the shell thickness. A maximum enhancement factor of 5.5 for total emission is achieved by the $10 \mathrm{~nm}$ shell Au-CS UCNPs. This maximum enhancement factor at a separation of $10 \mathrm{~nm}$ is consistent with the simulation results in Liu's work. ${ }^{38}$ These simulations ascribe the total enhancement $\left(f_{\text {tot }}\right)$ from the $\mathrm{Au}$ nanoparticle to be a product of the excitation enhancement from the local $E$-field intensity $(E)$ and the emission enhancement from the increased Purcell factor $(F)$ plus antenna efficiency $\left(\eta_{\mathrm{a}}\right)$. As $\eta_{\mathrm{a}}$ is related to the initial quantum efficiency $\left(\eta_{0}\right)$ of the UCNPs, $f_{\text {tot }}$ from the Au nanoparticle is highly dependent on the $\eta_{0}$ of the UCNPs, with a higher $\eta_{0}$ resulting in substantially lower total enhancement factors, i.e. $\eta_{0}=0.1 \%$ and $f_{\text {tot }}=30$ vs. $\eta_{0}=10.0 \%$ and $f_{\text {tot }}=5$. The coating of an inert shell onto a UCNP increases the quantum efficiency by reducing surface defects, ${ }^{6,8}$ so does the annealing of certain UCNPs by reducing internal defects. ${ }^{20}$ In this work, both processes are applied cooperatively to the UCNPs. Simulations of the initial quantum efficiency attenuation to the internal defect density in Fig. S4(B) $\dagger$ show that the $\eta_{0}$ of UCNPs is highly dependent on the density of internal defects. This explains the modest total enhancement factor of 5.5 to be a value approaching the highest feasible factor achievable for the already highly efficient annealed CS UCNPs.

To further verify the surface plasmonic effect of Au nanoparticles on the CS UCNPs, we compared the lifetime of the single CS UCNPs and Au-CS UCNPs. The CS UCNPs display in Fig. 5(A) a common trend of increased lifetime with increasing shell thickness due to the nature of the lifetime of the $\mathrm{Yb}^{3+}$ ${ }^{2} \mathrm{~F}_{5 / 2}$ energy level increasing continuously with the inert shell thickness as non-radiative surface-quenching processes become passivated more effectively. This increased $\mathrm{Yb}^{3+}{ }^{2} \mathrm{~F}_{5 / 2}$
A

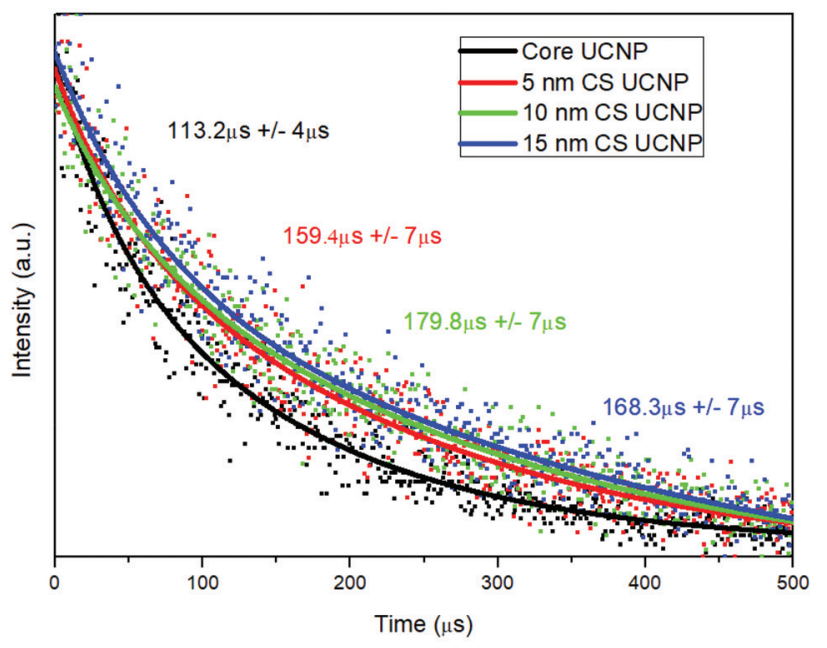

B

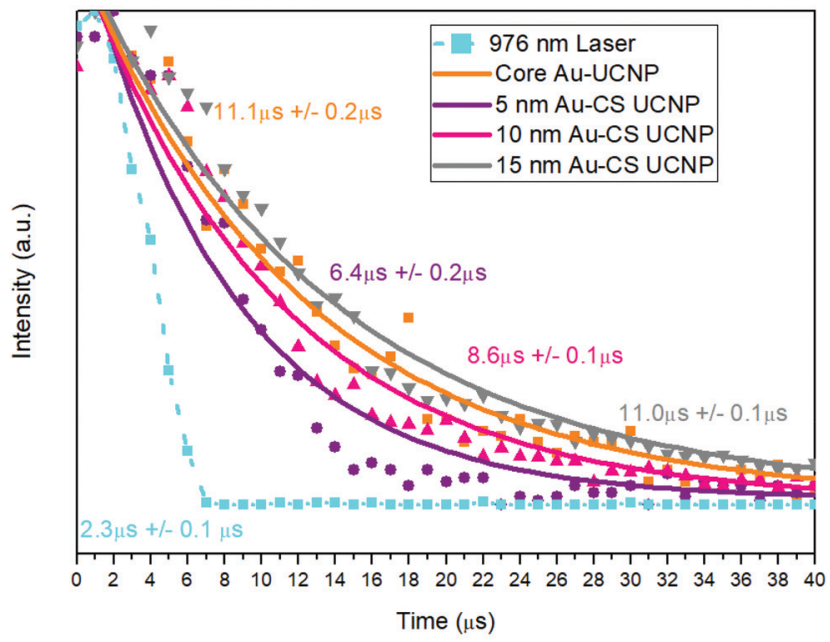

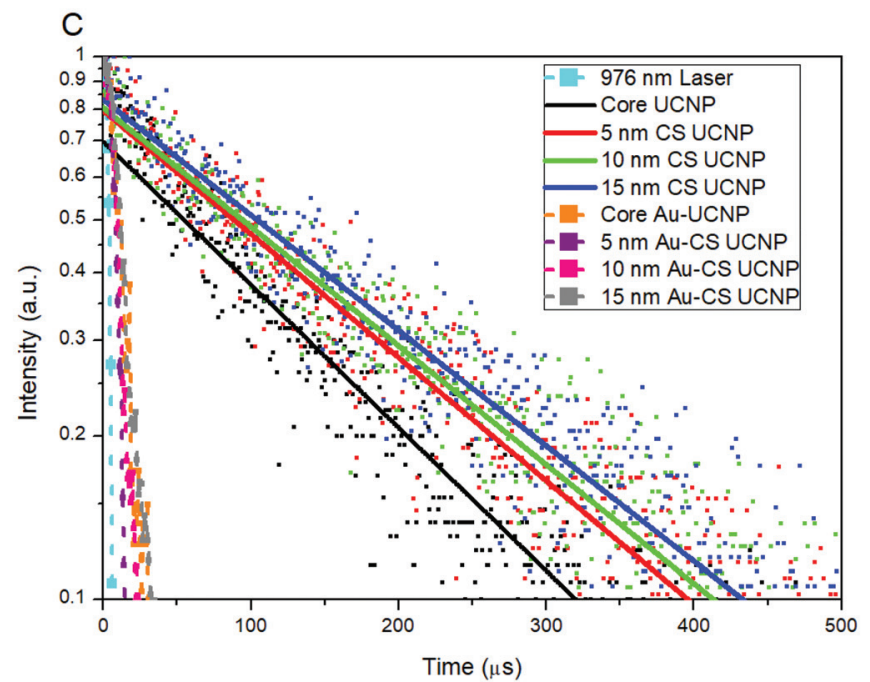

Fig. 5 Single nanoparticle lifetime measurement recorded for CS UCNP (A), Au-CS UCNP (B) and both single CS UCNP and single Au-CS UCNP on a semi-log plot (C). The laser power was fixed at $20 \mathrm{~mW}$, which was measured at the objective back aperture. The luminescence lifetime of the CS UCNPs decreases by more than an order of magnitude after decorating with dewetted Au nanoparticles. 
lifetime allows more time for the excitation energy to migrate through the nanocrystal, increasing the probability of energy transfer to an $\mathrm{Er}^{3+} \cdot{ }^{31}$ For all Au-CS UCNPs, a significant reduction in total lifetime was observed as shown in Fig. 5(B). The total lifetime of the $\mathrm{Au}-\mathrm{CS}$ UCNPs was reduced at a maximum by a factor of 25 , and this magnitude is clearly shown in Fig. 5(C). This approached the same magnitude as the lifetime of the laser used in the system measured at $2.3 \mu \mathrm{s}$ to verify the sensitivity of the system.

The decrease in emission lifetime for all Au-CS UCNPs is significant being among the highest recorded for plasmonic coupling $^{39,40}$ demonstrating the competition between two processes occurring, the Purcell effect and quenching from Förster-type energy transfer (FRET). ${ }^{41}$ The first process is the Purcell effect, which can increase the radiative recombination rate of an emitter by increasing the local density of transition states, thereby enhancing the emission intensity and reducing the emission lifetime. For upconversion emitters, e.g. $\mathrm{Er}^{3+}$, the intrinsic non-radiative recombination rate is much faster than the radiative decay rate, ${ }^{42}$ thus the change in the total lifetime of the emitter is dominated by the change in its radiative rate. Therefore, the total lifetime of Au-CS UCNPs is dramatically reduced, as its emission wavelength matches with the plasmonic resonance of the Au nanoparticles as shown in Fig. 3(A), which is consistent with previous research. ${ }^{43}$

Förster-type energy transfer (FRET) is the other process where the carriers on the emitter's energy level directly interact with the Au nanoparticles, leading to a reduction in both the emission intensity and lifetime through increasing the nonradiative rate. FRET is still significant when the effective separation distance between the dewetted Au nanoparticles and $\mathrm{Er}^{3+}$ emitters is below $15 \mathrm{~nm}$ (ref. 38), especially for the Au-CS UCNPs due to the direct contact of Au nanoparticles with the surface of the CS UCNP NaYF 4 host material.

The competition between the Purcell effect and FRET has been explored in the Au-CS UCNPs through the lifetime measurements. As a result of the competition, the lifetime of $\mathrm{Au}$-UCNPs $(11 \mu \mathrm{s})$ is longer than that of the Au-CS UCNPs with a shell thickness of 5, 10 and $15 \mathrm{~nm}$, as shown in Fig. 5 (B). As the shell thickness is increased to $15 \mathrm{~nm}$, the influence of FRET starts to diminish; however, the lifetime of the Au CS UCNPs is still an order of magnitude lower than the CS UCNPS lifetime, indicating that the Purcell effect dominates over the FRET. Further evidence of the dominant role of the Purcell effect is the decreased intensity in Fig. 4(I) and the increased lifetime in Fig. 5(B) and (C), as the shell thickness is increased beyond $10 \mathrm{~nm}$ on the Au-CS UCNPs. The thicker shell will reduce the Purcell effect, thereby reducing the radiative recombination rate, which leads to a decrease in intensity and an increase in lifetime.

\section{Conclusion}

The novel combination of CS UCNP synthesis, thermal annealing and dewetting of $\mathrm{Au}$ explored in this work produces $\mathrm{Au}-\mathrm{CS}$
UCNPs that exhibit the optimized plasmonic enhanced single particle upconversion emission. This enhancement is strongly dependent upon the thickness of the inert shell with a maximum enhancement factor of 5.5 in total emission intensity achieved by the $10 \mathrm{~nm}$ shell Au-CS UCNPs. The design and integrated method of fabrication of these Au-CS UCNPS represents a new direction in the research on the plasmonic enhancement of upconversion emission.

\section{Conflicts of interest}

The authors declare no competing financial interest.

\section{Acknowledgements}

XPS and NEXAFS measurements were undertaken on the Soft X-ray Spectroscopy beamline at the Australian Synchrotron. We wish to acknowledge the technical assistance of Drs Bruce Cowie and Anton Tadich. This project was primarily supported by the Australian Government Research Training Program Scholarship (Christian Clarke) and China Scholarship Council CSC scholarships (Chaohao Chen: No. 201607950009 and Yongtao Liu: No. 201607950010).

\section{References}

1 F. Auzel, Chem. Rev., 2004, 104, 139-174.

2 S. Gai, C. Li, P. Yang and J. Lin, Chem. Rev., 2013, 114, 2343-2389.

3 M. Nyk, R. Kumar, T. Y. Ohulchanskyy, E. J. Bergey and P. N. Prasad, Nano Lett., 2008, 8, 3834-3838.

4 L. Cheng, K. Yang, Y. Li, X. Zeng, M. Shao, S.-T. Lee and Z. Liu, Biomaterials, 2012, 33, 2215-2222.

5 L. Wang, R. Yan, Z. Huo, L. Wang, J. Zeng, J. Bao, X. Wang, Q. Peng and Y. Li, Angew. Chem., Int. Ed., 2005, 44, 60546057.

6 J. C. Goldschmidt and S. Fischer, Adv. Opt. Mater., 2015, 3, 510-535.

7 M. Pokhrel, A. Kumar Gangadharan and D. K. Sardar, Mater. Lett., 2013, 99, 86-89.

8 J.-C. Boyer and F. C. Van Veggel, Nanoscale, 2010, 2, 14171419.

9 S. Han, R. Deng, X. Xie and X. Liu, Angew. Chem., Int. Ed., 2014, 53, 11702-11715.

10 S. Fischer, F. Hallermann, T. Eichelkraut, G. von Plessen, K. W. Krämer, D. Biner, H. Steinkemper, M. Hermle and J. C. Goldschmidt, Opt. Express, 2012, 20, 271-282.

11 S. Fischer, D. Kumar, F. Hallermann, G. von Plessen and J. C. Goldschmidt, Opt. Express, 2016, 24, A460-A475.

12 S. Schietinger, T. Aichele, H.-Q. Wang, T. Nann and O. Benson, Nano Lett., 2009, 10, 134-138.

13 W. Feng, L.-D. Sun and C.-H. Yan, Chem. Commun., 2009, 4393-4395. 
14 H. Zhang, D. Xu, Y. Huang and X. Duan, Chem. Commun., 2011, 47, 979-981.

15 H. P. Paudel, L. Zhong, K. Bayat, M. F. Baroughi, S. Smith, C. Lin, C. Jiang, M. T. Berry and P. S. May, J. Phys. Chem. C, 2011, 115, 19028-19036.

16 C. M. Müller and R. Spolenak, Acta Mater., 2010, 58, 60356045.

17 R. Gupta, M. Dyer and W. Weimer, J. Appl. Phys., 2002, 92, 5264-5271.

18 C. Worsch, M. Kracker, W. Wisniewski and C. Rüssel, Thin Solid Films, 2012, 520, 4941-4946.

19 M. Kracker, C. Worsch, C. Bocker and C. Rüssel, Thin Solid Films, 2013, 539, 47-54.

20 N. C. Dyck, F. C. van Veggel and G. P. Demopoulos, ACS Appl. Mater. Interfaces, 2013, 5, 11661-11667.

21 F. Zhang, G. B. Braun, Y. Shi, Y. Zhang, X. Sun, N. O. Reich, D. Zhao and G. Stucky, J. Am. Chem. Soc., 2010, 132, 28502851.

22 W. Ge, X. Zhang, M. Liu, Z. Lei, R. Knize and Y. Lu, Theranostics, 2013, 3, 282-288.

23 C. Zhang and J. Y. Lee, J. Phys. Chem. C, 2013, 117, 1525315259.

24 L. L. Li, R. Zhang, L. Yin, K. Zheng, W. Qin, P. R. Selvin and Y. Lu, Angew. Chem., Int. Ed., 2012, 51, 6121-6125.

25 P. Kannan, F. Abdul Rahim, R. Chen, X. Teng, L. Huang, H. Sun and D.-H. Kim, ACS Appl. Mater. Interfaces, 2013, 5, 3508-3513.

26 Z. Wang, W. Gao, R. Wang, J. Shao, Q. Han, C. Wang, J. Zhang, T. Zhang, J. Dong and H. Zheng, Mater. Res. Bull., 2016, 83, 515-521.

27 J. Shen, Z. Li, Y. Chen, X. Chen, Y. Chen, Z. Sun and S. Huang, Appl. Surf. Sci., 2013, 270, 712-717.

28 X. Li, D. Shen, J. Yang, C. Yao, R. Che, F. Zhang and D. Zhao, Chem. Mater., 2012, 25, 106-112.
29 N. J. Johnson, A. Korinek, C. Dong and F. C. van Veggel, J. Am. Chem. Soc., 2012, 134, 11068-11071.

30 D. Liu, X. Xu, Y. Du, X. Qin, Y. Zhang, C. Ma, S. Wen, W. Ren, E. M. Goldys and J. A. Piper, Nat. Commun., 2016, 7, 10254.

31 S. Fischer, N. D. Bronstein, J. K. Swabeck, E. M. Chan and A. P. Alivisatos, Nano Lett., 2016, 16, 7241-7247.

32 T. L. Barr and S. Seal, J. Vac. Sci. Technol., 1995, 13, 12391246.

33 G. Yi, H. Lu, S. Zhao, Y. Ge, W. Yang, D. Chen and L.-H. Guo, Nano Lett., 2004, 4, 2191-2196.

34 H. Assaaoudi, G.-B. Shan, N. Dyck and G. P. Demopoulos, CrystEngComm, 2013, 15, 4739-4746.

35 X. Xu, C. Clarke, C. Ma, G. Casillas, M. Das, M. Guan, D. Liu, L. Wang, A. Tadich and Y. Du, Nanoscale, 2017, 9, 7719-7726.

36 C. Ma, X. Xu, F. Wang, Z. Zhou, D. Liu, J. Zhao, M. Guan, C. I. Lang and D. Jin, Nano Lett., 2017, 17, 28582864.

37 C. Ma, X. Xu, F. Wang, Z. Zhou, S. Wen, D. Liu, J. Fang, C. I. Lang and D. Jin, J. Phys. Chem. Lett., 2016, 7, 32523258.

38 X. Liu and D. Y. Lei, Sci. Rep., 2015, 5, 15235.

39 D. M. Wu, A. García-Etxarri, A. Salleo and J. A. Dionne, J. Phys. Chem. Lett., 2014, 5, 4020-4031.

40 W. Zhang, F. Ding and S. Y. Chou, Adv. Mater., 2012, 24, OP236-OP241.

41 W. Park, D. Lu and S. Ahn, Chem. Soc. Rev., 2015, 44, 29402962.

42 M. Saboktakin, X. Ye, S. J. Oh, S.-H. Hong, A. T. Fafarman, U. K. Chettiar, N. Engheta, C. B. Murray and C. R. Kagan, ACS Nano, 2012, 6, 8758-8766.

43 D. Lu, S. K. Cho, S. Ahn, L. Brun, C. J. Summers and W. Park, ACS Nano, 2014, 8, 7780-7792. 